\title{
Reconocimiento e identidad en torno a la profesión del diseñador industrial ${ }^{1}$
}

\section{María Eugenia Correa ${ }^{2}$}

\author{
Categoría: Artículo \\ Fecha de recepción: 28 de mayo de 2012 \\ Fecha de aprobación: 12 de julio de 2012
}

\begin{abstract}
Resumen
En las últimas décadas, la disciplina del Diseño Industrial ha alcanzado mayor relevancia, tantoen la Argentina comoen el contextolatinoamericano en general. Este trabajo busca, en este sentido, analizar la figura del diseñador industrial a la luz de la tensión devenida de la desarticulación generada entre su formación y la posterior inserción laboral. Instancias que vuelven compleja la construcción de una identidad profesional definida, así como el alcance de un reconocimiento consolidado en el mercado y en la sociedad. Esta figura profesional deviene contradictoria y difusa al encontrarse sustentada históricamente en un discurso abocado a la producción industrial, pero que en la experiencia profesional "real" construye sus trayectorias en áreas diversas de inserción y acción.

El abordaje metodológico es de índole cualitativa, y la técnica de producción de datos utilizada es la entrevista, a fin de reconstruir las experiencias laborales y perfiles profesionales de los diseñadores industriales entrevistados, desde sus propias percepciones.
\end{abstract}

Palabras clave: Diseño industrial - reconocimiento - identidad formación profesional - inserción laboral.

\begin{abstract}
In the last decades, the discipline of Industrial Design had become more relevant, in Argentina as in the Latin-American context as well. In this sense, this work proposes to analyze this figure at the light of the tension emerged by the disarticulation between formation and the labor insertion.
\end{abstract}

1 Este trabajo forma parte de la tesis doctoral titulada "Entre la industria y la autogestión. Análisis de la inserción laboral de los diseñadores industriales de la Universidad de Buenos Aires (1990-2010)", en la cual se analiza el campo de desarrollo profesional de estos actores, a la luz de su formación obtenida.

2 Doctora en Ciencias Sociales (Universidad de Buenos Aires), Magíster en Sociología de la Cultura (Universidad de San Martín) y Licenciada en Sociología (Universidad de Buenos Aires). Becaria posdoctoral del Consejo Nacional de Investigaciones Científicas y Técnicas (CONICET), con sede en el Instituto de Investigaciones Gino Germani, Facultad de Ciencias Sociales, UBA. eugeniacorrea@ sociales.uba.ar 
Instances that turn into complex the construction of a defined professional identity and recognition consolidated in the market and society. This professional figure becomes diffused by being sustained historically in an industrial-production-discourse but in the 'real' professional experience it constructs trajectories over diverse spaces of action and insertion.

The methodological boarding is based on a qualitative research, and the technique of data production used is the interview, in a way of reconstructing the labor experiences and professional profiles according to the perceptions of the interviewed industrial designers.

Key words: Industrial design - recognition - identity - professional formation - labor insertion.

\section{Introducción}

La actividad del diseño, y específicamente del diseño industrial, ha alcanzado en las últimas décadas una mayor relevancia tanto en el plano económico, como social y cultural. El crecimiento de las denominadas Industrias Creativas ha puesto de relieve la importancia de las mismas, entre las cuales el diseño presta un servicio de valor en la producción de bienes, dando cuenta del aporte de la economía 'creativa' en el sistema productivo.

Ahora bien, con respecto a la actividad específica de los diseñadores, a la acción de los sujetos que actúan delineando y construyendo los bienes que consumimos a diario, poco se ha dicho sobre esta figura, sumergiéndola de alguna manera en la invisibilidad. Es así como este trabajo se propone, ante cierto vacío en la literatura de las Ciencias Sociales en torno a esta práctica específica ${ }^{3}$, dar cuenta de dos cuestiones centrales en la constitución de esta figura profesional: el reconocimiento y la identidad.

3 Si bien hablamos del Diseño, o específicamente del Diseño industrial, en tanto práctica, es importante pensarla asimismo como disciplina constituida en torno a saberes múltiples como la Arquitectura Moderna, las Artes o mismo la técnica, campos de saberes que han permeado la configuración profesional del diseñador industrial desde mediados del siglo $\mathrm{XX}$, a través de dos escuelas fundacionales de esta disciplina, a saber: la Bauhaus, creada en 1919 en Weimar, Alemania, bajo la dirección de Walter Gropius, y la Hochschule für Gestaltung de Ulm, fundada en el año 1953 por Inge Aicher-Scholl, Otl Aicher y Max Bill, en el mismo país. 
La constitución de una disciplina basada en saberes diversos vuelve la mirada sobre un profesional formado ante un abanico de posibilidades de acción en las cuales desempeñarse. Cuestión que ciertamente complejiza el análisis de esta figura, cuya identidad se construye en la oscilación entre un postulado de formación que aún obedece a un desempeño abocado a la industria, y una inserción profesional diversa de acuerdo a la oferta cada vez mayor que se le presenta a este profesional en el mercado.

Este trabajo busca analizar justamente esta tensión devenida de la desarticulación generada entre formación profesional e inserción laboral, lo cual vuelve compleja la construcción de una identidad consolidada para el caso del diseñador industrial.

\section{La construcción del objeto: abordaje metodológico}

En relación al análisis de la inserción laboral de los diseñadores industriales, así como de sus perfiles e identidades profesionales, es preciso mencionar el corpus obtenido a partir de las entrevistas realizadas, en el marco de un abordaje metodológico cualitativo, a 25 diseñadores industriales egresados de la Facultad de Arquitectura, Diseño y Urbanismo de la Universidad de Buenos Aires (FADU-UBA). A partir de esta técnica se propuso comprender los discursos y prácticas de estos profesionales, en relación a cuestiones relevantes a los fines de la investigación, como ser, su modalidad de participación en el espacio productivo, su visión de integración en el mercado laboral, en la industria argentina, sus experiencias en torno a la formación profesional y sus percepciones y visiones acerca de sí mismos como profesionales.

En relación a las entrevistas en profundidad realizadas ${ }^{4}$, las mismas fueron desarrolladas en función de una guía de pautas construida en una primera instancia de salida a campo. Posteriormente este instrumento fue modificado a partir de la apa-

4 Cabe destacar que las mismas fueron realizadas en el período comprendido entre noviembre de 2010 y julio del 2011. 
rición de temas emergentes al realizar las primeras entrevistas. Esta guía se constituyó en base a diversos ejes de análisis, los cuales buscaron recuperar la visión de los entrevistados en torno a temas como: sus motivaciones con respecto a la elección de la carrera, sus percepciones con respecto a la formación educativa en la Universidad, sus diversas experiencias laborales -a fin de reconstruir sus propias trayectorias-, sus percepciones sobre el mayor o menor grado de conocimiento y de reconocimiento de la profesión y de sí mismos como profesionales en relación al mercado, la industria y la sociedad en general, así como sus expectativas o perspectivas con respecto a sus desarrollos profesionales, y mismo en relación al servicio de diseño en el país.

Cabe destacar que la selección de casos fue realizada en función de un muestreo teórico, que tuvo su inicio en un muestreo de tipo intencional, pero que se continuó a partir de los datos que se iban obteniendo en el proceso de relevamiento. Esto dio lugar a una búsqueda de casos que tuviera por fin alcanzar una exhaustividad en términos teóricos, esto es, que permitiera captar los casos coincidentes, así como aquellos divergentes, en cuanto a perfiles y trayectorias de los profesionales. Entonces, con respecto a la muestra final, la misma quedó conformada de la siguiente manera: cuatro diseñadores orientados a la participación en la industria, trece diseñadores con estudio de diseño independiente, un diseñador free-lance, dos diseñadores autoproductores y cinco diseñadores docentes-investigadores. De este modo, la muestra se conformó en torno a 25 entrevistados que abarcaron el 'abanico' de perfiles profesionales en relación al Diseño Industrial, reflejando, y tal el propósito del proceso de selección mismo, la diversidad de acción que caracteriza a la profesión.

\section{Acerca del concepto de Diseño y su configuración como campo de 'saberes' diversos}

Al definir la actividad de Diseño, la misma puede ser planteada en tanto práctica creadora de formas cuya lógica de producción 
se inscribe en un proceso de "proyectación" de una idea. Esto es, "definido como la concepción y planificación de todos los productos elaborados por el hombre, el diseño se puede considerar un instrumento para mejorar la calidad de vida" (Fiell y Fiell, 2001: 4). Ahora, si se piensa en la definición clásica del término 'diseño' podemos decir que el mismo remite a una práctica que tiene como objeto la creación de formas que habrán de realizarse posteriormente mediante procedimientos manuales o mecánicos (Diccionario Espasa-Calpe, 1986: 401-402). Su fin, es, ante todo, atender los aspectos funcionales de los objetos, así como sus condiciones de uso. Al mismo tiempo, según el diseñador industrial John Heskett, el diseño remite a "la capacidad humana para dar forma a nuestro entorno, para servir a nuestras necesidades y dar sentido a nuestras vidas" (2005: 7). De este modo, el diseño nace con el fin de dar forma a los objetos que son incorporados a la vida cotidiana a fin de satisfacer necesidades inherentes a la misma. Pero en este acto mismo de "dar forma" es que confluyen múltiples discursos provenientes de campos diversos.

Esto es, la configuración disciplinar del Diseño se ha construido en un marco dinámico de definiciones y redefiniciones de esta práctica, inicialmente en torno a la forma y a la calidad de los productos industriales, a la dicotomía artesanal-industrial, cuestiones que luego se continuaron en discusiones sobre la preeminencia o no de la funcionalidad o la formalidad en los productos, así como el encuentro o desencuentro con las artes, lo cual acercaba o alejaba, por cierto, a la figura del diseñador de la del artista-artesano. Hablamos de cuestiones que contribuyeron a sentar las bases del Diseño Industrial como profesión, y del diseñador industrial como profesional atravesado por diferentes saberes, tanto técnicos, tecnológicos, industriales, como artísticos, semánticos, o morfológicos.

El diseñador como profesional es formado, entonces, sobre una base múltiple de acción, en la medida que requiere de herramientas diversas que se vuelven necesarias para desarrollar su labor. Esto, porque el diseño constituye en sí un eslabón importante en la cadena productiva, con lo cual el profesional debe 
articular diferentes instancias que atañen tanto a la forma, el concepto y la función de los productos a desarrollar, como a las tecnologías y los materiales a través de los cuales serán producidos, así como cuestiones vinculadas a la comercialización y el consumo de los mismos, atendiendo las demandas y necesidades del público consumidor.

Es decir, que la construcción de la disciplina vuelve al diseñador una figura compleja en cuanto a la diversidad inherente a la formación obtenida, la cual lo habilita a desarrollar actividades diferentes según las demandas del mercado y del contexto económico, productivo, social y cultural en que se inserta. En este sentido, dar cuenta de cuestiones tales como el reconocimiento y la identidad profesional de los diseñadores industriales remite a instancias dinámicas, en constante redefinición, sea por las cambiantes prácticas profesionales, adaptadas a contextos de coyuntura económica, o por la misma redefinición de la disciplina en el ámbito universitario, dada la necesidad de habilitar una profesión basada, de alguna forma, en la diversidad de acción de sus profesionales, acompañando los múltiples requerimientos del mercado y de la sociedad.

\section{Construir identidad en la diversidad}

La conformación de perfiles profesionales diferenciados en torno a esta disciplina se inscribe en una formación, y una posterior inserción, que apela a la diversidad de acción. ${ }^{5}$ Es esta misma

5 Esta diversidad de acción a la que aludo, evidencia la participación de estos diseñadores en áreas diversas, tales como: el armado y diseño de 'stands', de mobiliario comercial (denominado POP, o punto de venta); diseño de objetos de uso cotidiano; de vehículos o medios de transporte; de maquinarias de uso agrícola o industrial; diseño de packaging o de envases, de render (entendido como la diagramación de una animación en $3 \mathrm{D}$, que, en tanto representación, simula ambientes y estructuras físicas verosímiles, utilizado, por ejemplo, en la visualización de proyectos arquitectónicos de viviendas, edificios, parques, y demás construcciones que se deseen proyectar); branding (diseño de marca corporativa); diseño de señalética, entre otros servicios y tareas realizadas. 
diversidad la que incide en la propia configuración de la identidad profesional del diseñador industrial, construida tanto en relación a elementos subjetivos como objetivos que permean al sujeto. Esto es, tanto la propia biografía como su entorno socialinstitucional se entrelazan en la definición identitaria del diseñador, dando lugar a la construcción de su identidad profesional. En este sentido, podemos decir que:

La identidad no es otra cosa que el resultado a la vez estable y provisorio, individual y colectivo, subjetivo y objetivo, biográfico y estructural, de los diversos procesos de socialización que, conjuntamente, construyen los individuos y definen las instituciones. (Dubar, 2001: 109).

Esto es, la identidad, de acuerdo a este autor (2002), se conforma en relación a dos dimensiones, una personal o biográfica -la identidad para sí- y una relacional -la identidad para otro-, poniendo en juego una articulación de los mencionados elementos subjetivos y objetivos que tienen lugar en el proceso de construcción identitaria. Es así que:

El sujeto construye su identidad a partir de una transacción interna al individuo, delineando "qué tipo de persona uno quiere ser" en correspondencia con su biografía; y una transacción externa entre el individuo y las instituciones y grupos a los que pertenece, a través de la cual se perfila "qué tipo de persona uno es", y con ello a qué definición oficial corresponde. De esta manera y a través del conjunto de representaciones sociales que conforman, por un lado, el acto de pertenencia, y por otro, el de atribución, el sujeto edifica en una misma identidad dos dimensiones de sí: la identidad para sí y la identidad para otro. Ambos aspectos de la identidad son inseparables y se construyen en un proceso continuo en el que el sujeto y su entorno se entre-trascienden y determinan. (Dubar, 2000b, en Longo, 2003: 5). 
En relación a esta puesta en juego de definiciones establecidas por los otros, definiciones "oficiales" que delinean el tipo de persona que somos, articuladas con deseos y aspiraciones propias en función de lo que queremos ser, es que se construye la base de la identidad profesional. Con lo cual, podemos plantear que:

La identidad profesional dependerá de la definición desde sí mismo y desde la otredad en cuanto a componentes como las competencias, el estatus, la carrera posible, la construcción de proyectos y las aspiraciones. Este ejercicio construirá una "identidad profesional de base" que permitirá una proyección de sí para el porvenir y que por lo tanto orientará la trayectoria de empleo y la lógica de aprendizaje o formación. Es decir, que "no se trata sólo de elegir un oficio o profesión o de obtener un diploma, sino de la construcción personal de una estrategia identitaria que pone en juego la imagen del $Y_{0}$, la apreciación de capacidades y la realización de deseos". (Dubar, 2000, en Machuca Barbosa, 2008: 53).

La constitución de la identidad profesional es dinámica, en tanto resultado de un proceso cambiante atravesado por instancias diversas que interactúan, dando lugar a una identificación del egresado con un grupo de pertenencia, así como a una diferenciación con respecto a otros grupos profesionales:

Las identidades profesionales son entendidas como formas identitarias en el sentido de configuraciones Yo-Nosotros que se localizan en el ámbito de las actividades de trabajo remuneradas. Son formas socialmente reconocidas de identificarse mutuamente en el ámbito del trabajo y del empleo, en el sentido de relaciones sociales y de trayectorias biográficas de vida laboral (Dubar, 2002:113). (...) Son el complejo de representaciones profesionales adquiridas en el proceso de interacción durante su formación profesional que permiten identificarse o diferen- 
ciarse de otros grupos profesionales en cuanto a su ser y quehacer profesional. (Machuca Barbosa, 2008: 53).

La identidad se construye, entonces, sobre esta base de la diferencia, así como de la pertenencia, en el sentido de que ambas instancias contribuyen en igual medida a su conformación. En relación a esto, y principalmente a la instancia constitutiva de la identidad profesional basada en el 'diferenciarse' respecto de otros grupos profesionales, una cuestión ciertamente significativa en torno a la profesión de los diseñadores industriales tiene que ver con su mentada vinculación con disciplinas como las Artes, la Arquitectura, o mismo la Ingeniería, al entrecruzarse en los procesos de configuración artística, proyectual o de producción industrial que las atañen. Esta cuestión incide, a su vez, en el reconocimiento social de los diseñadores, así como en el proceso de construcción de su identidad profesional, al identificarse con o diferenciarse de estos otros grupos profesionales, los cuales presentan cierta convergencia con su actividad laboral. Analizaremos en el próximo apartado esta cuestión.

\section{Diseñadores, entre ingenieros y arquitectos}

Ante la existencia de una diversidad de perfiles profesionales, la propia definición identitaria del diseñador industrial se vuelve ciertamente compleja. Esto, debido a que la misma tradicionalmente ha estado configurada bajo el supuesto -como mencionara un entrevistado, bajo el "postulado moderno europeo"- de ser concebido para diseñar productos industriales. Pero, ante la redefinición disciplinar, devenida en tanto formación con orientación hacia lo 'múltiple' dados los diversos requerimientos del mercado y la oscilante situación de la producción industrial, esta configuración identitaria, también se ha redefinido, adquiriendo una nueva significación, basada ahora en la conformación de un perfil profesional 'múltiple'.

A su vez, el entrecruzamiento con disciplinas cercanas, vinculadas a lo proyectual, interviene en el reconocimiento del dise- 
ñador como profesional, al volverlo una figura compleja, con una especificidad un tanto indefinida. No es solamente en relación a las diversas carreras de Diseño, que en sí ya dentro de este mismo campo se manifiesta la pluralidad, sino con respecto a la cercanía que presenta con el Arte, la Arquitectura, o mismo la Ingeniería. Disciplinas cercanas y fronterizas, que ante la amplitud y diversidad de acción profesional del diseñador, se entrecruzan con el campo del Diseño, volviéndolo una profesión -con una autonomía ciertamente reconocida, portadora de un cuerpo de saberes propios-, cuya práctica aún debe diferenciarse de aquellas disciplinas que la definieron y constituyeron desde sus inicios como tal.

De este modo, se evidencia una inserción laboral no sin conflictos, puesto que el diseñador debe llevar adelante su integración y participación en el mercado 'abriéndose camino' entre las mismas, esto es, entre profesiones legitimadas por saberes 'tradicionales' -la Arquitectura como disciplina pionera de la actividad proyectual en el país, la Ingeniería como referente de un saber legítimo vinculado a la producción industrial-, cuyas figuras profesionales en un punto reflejan mayor especificidad en sus desarrollos laborales, al mismo tiempo que se refleja sobre éstas un mayor conocimiento social, con respecto a la labor del diseñador:

“...Un ingeniero vos ya tenés una idea que tiene cierta preparación, como que ya su trabajo vale per se digamos, con lo que esta persona ya el título que tiene ya te implica que tiene tantos conocimientos. El diseñador como que es más una nebulosa lo que sabe en general. Y en la realidad también es un problema, es una nebulosa..." (Santiago, diseñador industrial, trabaja en empresa) $)^{6}$. “...Como que los empresarios conocen a los ingenieros, a los arquitectos, pero no saben lo que es un diseñador

6 Los nombres de los entrevistados han sido modificados para garantizar la confidencialidad de los mismos. 
industrial..." (Patricia, diseñadora industrial, autoproductora).

“...Para insertarnos laboralmente, el primer obstáculo que hay que sortear es justamente el social. El Diseño Industrial es una profesión que no se la conoce, nos confunden con decoradores, ingenieros y licenciados..." (Gustavo, diseñador industrial en estudio independiente).

A su vez, desde los propios diseñadores se imparte este discurso que 'privilegia' la labor de los ingenieros a la de los diseñadores industriales, en términos de calidad y especificidad en el desempeño laboral, tal como expresa este entrevistado, quien cuenta con un estudio de diseño junto a dos socios, y en relación al mismo plantea que:

“...Hace muy poquito empezamos con un nuevo modelo de estructura en donde vimos que necesitábamos tener una estructura más flexible, teníamos empleados en relación de dependencia y estamos tratando de migrar a contratar más especializados, a la persona que trabaja en el proyecto para mejorar la calidad y bajar los tiempos. Yo sé que si contrato un ingeniero específico en materiales resuelve eso, parece que me cobra más caro, pero lo resuelve en dos días y a una mejor calidad entregable y en menos tiempo. Entonces estamos un poco apuntando a eso." (Miguel, diseñador industrial en estudio independiente).

En relación a este mayor reconocimiento de los ingenieros $\mathrm{o}$ arquitectos, otro entrevistado expresa sus percepciones sobre esta cuestión, instalando discursivamente una incertidumbre acerca de la figura de los diseñadores industriales y su propia definición, cuestión que diverge ciertamente para el caso de los arquitectos e ingenieros:

- "...Porque aparte también hay una cuestión, digamos, como de... de reconocimiento. $\mathrm{O}$ sea, no somos arqui- 
tectos ni ingenieros... entonces es como... ¿qué somos?" (Daniel, diseñador industrial, trabaja en estudio independiente).

- “... Sí, aún hoy te confunden normalmente con ingeniero industrial..." (Raúl, diseñador industrial en estudio independiente).

En relación a estos relatos, dos cuestiones se vuelven visibles en la voz de los entrevistados, esto es: la identidad y el reconocimiento. Por un lado, se manifiesta la necesidad de diferenciarse discursivamente de profesionales cuyas disciplinas, si bien se aproximan al Diseño, al mismo tiempo se presentan como 'tradicionales' en el abanico de las profesiones, tanto para el caso del arquitecto como del ingeniero. Lo cual genera, por otro lado, un distanciamiento de este posicionamiento de reconocimiento en el que se encuentran las mismas, negadas discursivamente como identitarias, al afirmarse: "no somos arquitectos ni ingenieros".

Si pensamos la noción de diferenciación, esto es, la acción de diferenciarse de aquellos con los cuales no nos sentimos identificados, se establece una de las operaciones sobre las cuales se sostiene todo proceso de construcción identitaria. Esto es, en términos de Dubar:

La identidad no es lo que permanece necesariamente "idéntico", sino el resultado de una "identificación" contingente. Es el resultado de una doble operación lingüística: diferenciación y generalización. La primera es la que tiende a definir la diferencia, la que incide en la singularidad de algo o de alguien en relación con los otros: la identidad es la diferencia. La segunda es la que busca definir el nexo común a una serie de elementos diferentes de otros: la identidad es la pertenencia común. Estas dos operaciones están en el origen de la paradoja de la identidad: lo que hay de único es lo que hay de compartido. (2002: 11). 
En el pasaje al que hacen alusión los entrevistados (Raúl y Daniel) al diferenciarse claramente de los arquitectos e ingenieros, se refleja esta operación de diferenciación expresada por Dubar, la cual incide en la propia configuración identitaria. Esto es, se diferencian del otro en tanto que adquieren una identidad propia, pero al mismo tiempo se establece una diferencia en términos de reconocimiento dada por la especificidad de estas disciplinas devenidas 'tradicionales', en clara divergencia con la amplitud o diversidad del Diseño, en tanto disciplina 'liberal', tal como la plantea Chiapponi (1999).

La discursividad en la que se vuelve manifiesta esta diferencia remite a una construcción identitaria que se inserta en ámbitos específicos de producción. En este sentido, de acuerdo a Stuart Hall (2003), podemos pensar que:

Precisamente porque las identidades se construyen dentro del discurso y no fuera de él, debemos considerarlas producidas en ámbitos históricos e institucionales específicos en el interior de formaciones y prácticas discursivas específicas, mediante estrategias enunciativas específicas. (Hall, 2003: 6).

Al mismo tiempo que agrega:

Las identidades se construyen a través de la diferencia, no al margen de ella. Esto implica la admisión radicalmente perturbadora de que el significado «positivo» de cualquier término -y con ello su «identidad»-sólo puede construirse a través de la relación con el Otro, la relación con lo que él no es, con lo que justamente le falta, con lo que se ha denominado suafuera constitutivo.(Hall, 2003:6).

Es decir, que se instala discursivamente una cuestión específica: la supuesta falta de reconocimiento, desde la visión del entrevistado, al preguntarse "¿qué somos?", en oposición al reconocimiento social de la figura del arquitecto o del ingeniero, al mismo tiempo que se pone en juego una construcción de identi- 
dad a partir, justamente, de no identificarse como aquel otro que es socialmente reconocido. La identidad se nutre de estas diferencias, se construye en la misma diferencia con el otro -"a través de ella", como expresa Hall-, en este caso, en el no reconocerse como otro que es reconocido. Es así que:

Diferenciación, identificación y reconocimiento son momentos inseparables y articulaciones del proceso de construcción de identidad, y están situados en el seno de la experiencia conflictual y social de las relaciones humanas. (Sainsaulieu, 1988, en Longo, 2003: 7).

Lo positivo se instala en esta negación: la identidad es inherente a este reconocimiento de un no reconocimiento. Esto es, afirmativamente la identidad es dada a partir del reconocimiento:

El individuo participa en la definición de su identidad. Se podría decir que negocia con su entorno, pero no dispone de su plena voluntad. No es por casualidad que la época de la revolución expresivista vea el nacimiento de otro discurso, el del reconocimiento. Hablo del tema del reconocimiento por otro como condición de la identidad lograda. El individuo tiene necesidad, para ser él mismo, de ser reconocido. (...) No podríamos definirnos por nosotros mismos. Tenemos necesidad del concurso de los «otros significativos». (Taylor, 1999: 4).

La identidad se construye en función del reconocimiento del otro. Para el caso de los diseñadores, si bien los mismos son reconocidos como profesionales (tanto institucionalmente, por la Universidad, por sus clientes, por el mercado), cuestiones como: el desconocimiento que los sujetos mencionan que aún impera en torno a la profesión, o mejor dicho, con respecto a las competencias específicas del diseñador industrial, principalmente en el ámbito industrial (su supuesto espacio específico y legitimado de acción), lo cual conduce a una escasa incorporación o contratación de diseñadores en las empresas; la baja remuneración que perciben por las tareas realizadas -cuestión recurrente en las en- 
trevistas-; la confusión que aún impera con respecto a la figura de los ingenieros, sobre todo, los industriales; así como la mayor o menor vinculación con las disciplinas del Arte o la Arquitectura, indican que el reconocimiento de estos profesionales se ve inmerso en una contradicción. La contradicción que, sin dudas, connota esta situación pone en evidencia las consecuencias de una formación 'ampliada': beneficiosa en términos de inserción laboral, inespecífica al momento de delimitar la figura del diseñador como profesional. En este sentido, podemos pensar que se reconoce la profesión, la actividad del diseño, pero no concretamente las competencias de este profesional: la dificultad está en reconocer específicamente esta figura.

Ante esto, cabe la pregunta: ¿Incidiría una mayor especificidad profesional en un mayor reconocimiento de los diseñadores? En un punto, esta redefinición disciplinar debe acentuarse, consolidarse, instalarse legítimamente en los discursos y prácticas que atañen a la disciplina y su formación, puesto que, dado que aún conviven discursos de tipo 'industrialista' con otros más abiertos y diversos en cuanto a la actividad profesional, los diseñadores, como consecuencia, se vuelven una figura vulnerable a los contextos diversos en que se insertan. Su identidad, entonces, deviene ciertamente compleja. En este sentido, tal como expresa Battistini (2009), evocando la noción de identidad de Cuche (2001), podemos reflexionar en torno a la siguiente cuestión:

Nuestros tiempos muestran, cada vez con mayor crudeza, la complejidad creciente de nuestras sociedades. Si la identidad es una construcción social, dicha complejidad, entonces, no puede serle ajena. Ningún grupo ni individuo está encerrado a priori en una identidad unidimensional. Es precisamente, su carácter fluctuante lo que hace que se preste a diversas interpretaciones. La identidad resulta difícil de delimitar y definir debido a su carácter multidimensional y dinámico. Esto es lo que le confiere su complejidad, pero es lo que le da flexibilidad. La identidad conoce así variaciones, se presta a 
reformulaciones y también a manipulaciones. (Cuche, 2001: 91-92, en Battistini, 2009: 2).

La figura del diseñador industrial deviene compleja, dada la diversidad de discursos que atraviesan a la misma disciplina, desde sus inicios como tal. Esta identidad que define al diseñador, tal como expresáramos anteriormente, se construye en función del sí mismo así como del reconocimiento del otro, de componentes subjetivos y objetivos que atraviesan la trayectoria del individuo, su instancia biográfica. La cuestión del reconocimiento en tanto profesionales se entremezcla discursivamente con el planteo de los posicionamientos que mantienen las figuras del ingeniero y del arquitecto en el escenario productivo, así como en el imaginario social. Ahora, ante la pregunta que se plantea el entrevistado, cuando expresa: "no somos arquitectos ni ingenieros... entonces es como ¿qué somos?" se pone en evidencia una grieta entre la identidad concebida 'para sí' y la identidad 'para el otro', al verse carente de identidad ante el no reconocimiento en tanto profesionales reconocidos, esto es, entre los arquitectos o ingenieros. Si el reconocimiento viene de la mano de pertenecer a una disciplina 'reconocida' socialmente, como la Arquitectura o la Ingeniería, la pregunta que surge es: ¿cómo volverse reconocido desde una disciplina cuyo reconocimiento social aún alberga tergiversaciones o confusiones acerca de la propia práctica? ¿Cómo ser reconocido si todavía la propia figura se presenta imprecisa, dada su complejidad y diversidad? En relación a esto, una entrevistada reflexiona:

“...Me parece que nos deja a nosotros medio en un lugar de autovendernos, pero ¿cómo te vendés? ¿Como qué?" (Sandra, diseñadora industrial en estudio independiente).

Si la entrevistada cuestiona el “¿Cómo te vendés? ¿Como qué?", es porque en cierta medida la indefinición de la figura no permite en algún punto identificar con claridad a este profesional en un rol o perfil determinado, con lo cual, ante una oferta impre- 
cisa -o lo suficientemente amplia-, tiene lugar una demanda escasamente definida. Esto, justamente, por la propia imprecisión inherente a la configuración de la disciplina:

"El Diseño Industrial es una disciplina, por supuesto, que tiene ribetes técnicos, pero no es una ciencia. Tiene mucha influencia, cada vez más, o por lo menos se le reconoce cada vez más, una cuestión humanística. Y depende de cómo cada uno lo tome, cada pequeño universo de diseñadores tiene una orientación u otra, y la enseñanza es también así y la historia ha sido así. Entonces es muy difícil decir, por ahí a nosotros nos resulta fácil decir, la Ingeniería siempre fue Ingeniería y la Medicina siempre fue Medicina..." (Entrevista a Ricardo Blanco).

Esto ciertamente se vincula a la ausencia de especialización en la carrera, o mismo, a la imprecisión en cuanto a la delimitación o definición de la disciplina -tal como sostiene una entrevistada, en el único punto en que se acuerda institucionalmente su precisión es en cuanto a que remite a una "práctica proyectual"-, denotando, así, la complejidad de la figura del diseñador, la encrucijada sobre la cual se construye la misma. Esta instancia incide en gran medida al momento de ser reconocido profesionalmente por el otro -como por ejemplo, por potenciales clientes, industriales de pequeñas y medianas empresas (PyME), entre otros-, al no saber exactamente qué sería o qué no sería propio o específico de la profesión, esto es, qué sería específicamente un diseñador industrial:

“...Cuesta o por ahí no está como ya trazado el camino, ¿no? Esto que decía, que por ahí hay como una falta de conocimiento, y entonces... Digo, entre la demanda y lo que nosotros hacemos hay por ahí un hueco. $\mathrm{O}$ sea, lo de la oferta es muy diversa, que lo hace muy difícil disfrazar a alguien de diseñador industrial y decir "bueno, éste es nuestro representante", ¿no? "Esto nos representa". Porque en realidad hay gente que es... Produce sus 
propios diseños y los vende en lugares como, digamos, donde se valora el valor estético que tiene el producto y demás, y otras que trabajan en una metalúrgica mejorando la... Digamos, mucho más cercanos a la Ingeniería. Nos definimos mucho también en función de a qué nos acercamos, que estén más cerca de un trabajo más de Ingeniería o más de Arquitectura o... Yo, por ejemplo, últimamente estoy mucho más vinculada al rubro de lo que es como de moda e indumentaria que industrial propiamente dicho." (Sandra, diseñadora industrial en estudio independiente).

En este pasaje se observa la dificultad de 'visibilizar' la figura precisa del diseñador, lo cual se ve reflejado cuando la entrevistada plantea que "se hace difícil disfrazar a alguien de diseñador", dejando en claro que no tiene, o no tendría, un rol ciertamente definido. A su vez, aparece nuevamente la proximidad a las disciplinas de Ingeniería o Arquitectura, cuando ella plantea: "nos definimos también en función de a qué nos acercamos", de modo que las mismas constituyen ciertos parámetros de definición identitaria. El 'qué somos', si se relaciona a disciplinas 'tradicionales' como las mencionadas, encuentra una respuesta más precisa, y en función de esto, la posibilidad de mayor reconocimiento. Ante esto, nos preguntamos: ¿la identidad del diseñador se construye sobre la base de la diferencia o de la proximidad con respecto a profesionales 'reconocidos' como el ingeniero o el arquitecto? Tal como mencionáramos en líneas anteriores, se supone una construcción identitaria sobre la diferencia con otros grupos profesionales, la operación de la diferenciación a la que hacía referencia Dubar, a partir de la negación del ser otro ("no somos arquitectos ni ingenieros"), pero a su vez, aquí se refleja una autodefinición de identidad al acercarse a este otro, si bien no desde una identificación con los mismos, tampoco los vuelve claramente diferenciados en cuanto a la actividad que realizan. 
En este sentido, se evidencia una contradicción en este mismo relato, manifestado claramente en relación a la grieta percibida en cuanto al propio sentido de pertenencia a un grupo definido, justamente, porque no se percibe un grupo propio de referencia que dé lugar a la operación de 'generalización' alcanzada por los otros grupos profesionales (arquitectos, ingenieros). Al no predominar un sentido de pertenencia a un colectivo profesional ('los diseñadores industriales'), tiene lugar esta proximidad o este acercamiento (autoasignado o dado 'desde afuera') a estos grupos profesionales. La falta de cohesión los conduce a buscarla, a percibirla, desde otro lugar. El conflicto que en un punto les genera la confusión con estos otros profesionales, mayormente reconocidos, se vuelve una instancia de identificación de sí mismos como próximos a estos, que actúa atenuando los efectos de la falta de generalización. En este sentido, podemos pensar que:

Las identidades se nutren constantemente de identificaciones que cimientan la subjetividad, y con ello construyen microscópicamente las prácticas de cada persona. Las fuentes significativas e identificatorias de las identidades pueden ser múltiples. Existe todo un abanico de discursos interpelantes que materializan actos permanentes de atribución a los sujetos. (...) Debido a la multiplicidad de interpelaciones, la identidad no se compone de identificaciones armónicas; está interceptada por prácticas y discursos diferentes o antagónicos. (Longo, 2003: 6).

La identidad, en tanto resultado de un proceso social dinámico, se construye sobre relaciones de identificación y diferenciación que suponen lazos divergentes, antagónicos, dotados de encuentros y desencuentros que convergen en el marco de la propia socialización, no sin conflictos mediante.

Cabe destacar que la proximidad o cercanía con estas disciplinas, o mismo su diferenciación, evidenciada en los relatos de los entrevistados, nos remite a sus propios planteos acerca del 
porqué de la elección de la carrera. En este sentido, es importante mencionar que gran parte de los entrevistados ha elegido estudiar Diseño Industrial puesto que contaban con la referencia de familiares, amigos o conocidos arquitectos o ingenieros, de modo que podemos pensar que, desde el momento mismo en que se vieron motivados o atraídos a empezar estos estudios, existía una mentada vinculación con alguna de estas disciplinas:

“...Mirá, mi viejo había estudiado Arquitectura y tengo varios familiares que son ingenieros. Y qué sé yo, del tema de dibujar, si bien a mí no me salía muy bien, era como que siempre estábamos en una familia con todo el tema de las artes, y desarrollarse en eso, está bien visto así que..." (Julio, docente universitario y diseñador en estudio independiente).

“...Mi viejo es arquitecto, tal vez por ahí entré... La verdad que no recuerdo bien por qué me interesó el Diseño. Supongo que sí, la cercanía con mi viejo..." (Daniela, becaria $\mathrm{UBACyT}^{7}$ y docente universitaria FADU-UBA ${ }^{8}$ ).

“...Bueno, vengo de dos padres ingenieros, uno Ingeniería Química y el otro Ingeniería Industrial, así que... mi familia... yo también ¿eh?... como que también te facilita..., no sé si te facilita, pero hay lógicas..." (Claudia, trabaja en $\left.\mathrm{INTI}^{9}\right)$.

"...Porque siempre fue, todo lo que tenga que ver con actividades plásticas, siempre tuve facilidad, mi papá es ingeniero industrial y para mí era una rama muy dura en cuanto a lo teórico y no veía que fuera por ahí. Y Arquitectura no me llamaba, era una escala que para mí

7 Beca de investigación otorgada por la Secretaría de Ciencia y Técnica de la Universidad de Buenos Aires.

8 Facultad de Arquitectura, Diseño y Urbanismo de la Universidad de Buenos Aires.

9 Instituto Nacional de Tecnología Industrial. 
nunca me llamó la atención. Y fui a una charla que daba Ricardo Blanco, no sé si todavía siguen dando, de orientación vocacional... y ese día daban Industrial, Gráfico e Indumentaria. Y yo dije "o es gráfica o es industria". Y me encantó la charla de Industrial y ahí decidí. Con la historia mía familiar de mi viejo industrial, qué sé yo, iba por ese lado, me sentía más cómodo..." (Jorge, diseñador free lance).

La herencia familiar, así como los referentes cercanos, se constituye como una dimensión significativa en la trayectoria profesional, específicamente en el momento de elección de la carrera y orientación a seguir en la formación de nivel superior.

Con respecto a esto, la relación con el Arte también es una referencia que, al igual que con la Arquitectura o la Ingeniería, emerge en el relato de los entrevistados, al plantearse las motivaciones que dieron lugar a pensar en elegir esta profesión:

“...En mi familia no había ningún diseñador, no había nada... no había ningún arquitecto... aunque lo que sí había en ese momento, que yo no entendía mucho la relación entre una cosa y la otra, era una licenciada en Artes que era mi mamá, licenciada en Historia del Arte... Mi viejo es psicólogo, y yo no entendía la relación en principio de Diseño Industrial con la Historia del Arte, después lo fui comprendiendo a medida que me fui adentrando en la carrera... y mi abuelo que fue mecánico, fue obrero toda la vida y que me llevó a mí a trabajar en la industria. En realidad yo creo que es un mix que tiene que ver con una historia familiar en ese sentido." (Pablo, docente en la carrera de Diseño Industrial-UBA y diseñador independiente en estudio propio).

“...Quería ser escultora, quería hacer arte. Yo tenía una pequeña formación en arte, por talleres particulares, había hecho cerámica, tanto en la primaria como después, en la secundaria, y bueno, me gustaba... pero bueno, 
dejé de hacer por falta de tiempo, ésta es una carrera que te consume mucho tiempo....Y considero que mi forma de ver el diseño tiene que ver con esa mirada artística." (Gabriela, autoproductora).

En relación a estas mentadas vinculaciones con las disciplinas del Arte o la Arquitectura, el hecho mismo de que las carreras de Diseño se hayan inscripto en el marco de la Facultad de Arquitectura -refiriendo al caso específico de la UBA- también le imprime relevancia, dado que el mismo ha marcado y ha sellado la relación significativa que el Diseño ha tenido, y tendrá, con esta disciplina proyectual. Este hecho tiene lugar a la luz de una corriente que fue predominante en aquel momento para las disciplinas proyectuales, y especialmente para la configuración de la Arquitectura Moderna y el Diseño Moderno -fundado sobre las bases del buen diseño-, como fue el funcionalismo.

\section{Diseñadores ¿industriales?}

Ahora bien, pensando nuevamente en la figura del diseñador y su constitución como resultado de una combinación de diversas áreas (artística, técnica, estética, semántica, morfológica, proyectual), podemos pensar que esta misma diversidad que, por un lado, brinda mayores herramientas y puede ser entendida como beneficiosa en términos de una formación 'completa', al mismo tiempo reduce el nivel de especialización en la formación, la cual debe ser obtenida posteriormente. Esto mismo, como he planteado, incide en la construcción de la identidad profesional, que ha sido redefinida en términos institucionales, al desarrollar, desde la propia Universidad, una formación 'ampliada', excediendo el marco laboral para el cual estaba inicialmente orientada, esto es, el diseño de productos industriales. En este punto, es importante mencionar que la demanda de 'autoproducir' los objetos al interior de la carrera provino de los mismos alumnos, la cual fue contemplada por la cátedra del Taller de Diseño Industrial -material central en la cursada- que le dio lugar y la gestionó como 
base de su formación al interior de la misma. Fueron los mismos alumnos quienes posteriormente iniciaron el circuito de diseño en Palermo ${ }^{10}$, dando mayor reconocimiento y visibilidad a los diseñadores, y sentando las bases de una redefinición identitaria, articulada entre lo meramente subjetivo -aquello que los alumnos querían para sí: ser diseñadores independientes- y aquello que institucionalmente fue redefinido: el perfil de diseñador de productos para la industria, ahora resignificado -o ampliado- a partir de esta modalidad -reposicionada en un contexto de crisis y dificultad de incorporación en las empresas- de diseñador 'autogestionado'. El contexto de crisis sin duda condujo a los alumnos a formarse como diseñadores independientes, ampliando así su marco de posibilidades de acción por fuera de la industria. Los diseñadores industriales devendrían entonces no industriales.

En este contexto de redefinición disciplinar, lo que se puso en juego fue la posibilidad de mostrarse en la escena pública, alcanzando un reconocimiento de esta figura profesional, o específicamente, del diseñador como 'autoemprendedor'. Esto es, si bien los entrevistados plantean que existe un mayor reconocimiento de la actividad del diseño, sin dudas se debe al mayor desarrollo de esta práctica en la última década. Tal como sostiene un entrevistado: “...a partir del 2001 hubo como un reconocimiento a cierta cultura del diseño que antes no había. El cambio fue ese...", con

10 Aquí hago referencia al desarrollo del barrio de Palermo, en la ciudad de Buenos Aires, en tanto 'polo de diseño', a partir de la instalación de locales y ferias destinadas a la difusión y comercialización de productos "de diseño". Estos emprendimientos vinculados al diseño de indumentaria y de objetos de uso cotidiano tuvieron lugar a partir de la denominada crisis del 2001 en la Argentina, momento a partir del cual la devaluación económica junto a políticas de impulso a la producción nacional, dieron lugar a una mayor circulación de bienes locales y al crecimiento de la actividad de diseño, principalmente a partir de la modalidad de diseño 'independiente' y 'de autor'. Estas prácticas, mayormente artesanales, o pseudo-artesanales, evidencian cierta distancia con respecto al tradicional diseño de productos industriales, para el cual son formados los estudiantes en la Universidad. Esta divergencia entre formación e inserción productiva encuentra, a partir de este momento de expansión del 'diseño independiente', una necesidad de reformulación de los lineamientos fundacionales de la disciplina, específicamente en contextos de fragilidad industrial. 
lo cual se refleja un antes y un después en cuestión de reconocimiento del diseño como actividad inserta en la sociedad. Pero, sin embargo, ante esta situación, aún la figura del diseñador industrial no resulta lo suficientemente reconocida y valorada, al punto de que aún persisten, como he mencionado anteriormente, los discursos que confunden el diseño industrial con la ingeniería, o que desconocen las competencias del diseñador en relación a la industria, lo cual plantea una lucha por la obtención de mayor reconocimiento y valoración de esta figura específica en términos profesionales. Pero, ante esto, cabe destacar que lo que sí denotó un reconocimiento de esta figura en la última década, ha sido la mayor actividad de tipo autogestionado realizada, la cual generó una mayor visibilidad de emprendimientos de tipo independiente en relación al diseño, como, por ejemplo, del 'diseño de autor'. Esto es, cuando los entrevistados refieren a un mayor reconocimiento del diseño a partir del 2001, están remitiéndose a esta situación, no justamente a la que da cuenta de la relación del diseñador con la industria. Es esta práctica autogestionada la que devino mayormente reconocida por la sociedad. En este sentido, Dubar expresa que:

Se puede establecer, de acuerdo a Sainsaulieu, la hipótesis de que la inversión privilegiada en un espacio de reconocimiento identitario depende estrechamente de la naturaleza de las relaciones de poder en ese espacio y del lugar que ahí ocupe el individuo y su grupo de pertenencia. ${ }^{11}$ (Dubar, 2000: 121).

A lo que luego agrega: "El espacio de reconocimiento de las identidades es inseparable de los espacios de legitimación de los saberes y competencias asociados a las identidades."12. Es decir que,

11 Texto original: “On peut donc, avec Sainsaulieu, faire l'hypothèse que l'investissement privilégié dans un espace de reconnaissance identitaire dépend étroitement de la nature des relations de pouvoir dans cet espace et de la place qu'y ocupe l'individu et son groupe d'appartenance." (Traducción de la autora).

12 Texto original: "L'espace de reconnaissance des identités est inséparable des 
si en la última década tuvo lugar un mayor reconocimiento de las prácticas de estos profesionales, vinculado a una mayor visibilidad de las mismas, es porque se plasmó en el campo una puesta en escena de competencias y saberes en juego que hicieron mayormente reconocible esta figura. Las mismas, justamente, se basaron en este despliegue de autoproducción en la escena pública.

El espacio de reconocimiento de las identidades, tal como expresa Dubar, es inseparable de los espacios de legitimación de los saberes y competencias. Con lo cual, ante esta redefinición de la disciplina que buscaba legitimar la demanda de diversificar los perfiles profesionales, se constituye un espacio de legitimación de estos nuevos saberes y competencias. Los mismos pasan, así, a conformar el nuevo marco de reconocimiento del diseñador, entendido no sólo como un mero diseñador de productos para la industria. El reconocimiento de este profesional se resignifica en este contexto, devenido de una crisis económica y social, al calor de diversas demandas sociales, y es por esta resignificación que el mismo pasa a ser socialmente reconocido.

En este sentido, es importante destacar que si bien la Facultad ha asumido la necesidad de reponer el ejercicio de la autoproducción como práctica profesional adaptada a un mercado que presenta una débil incorporación de diseñadores en la industria, aún la confluencia de discursos acerca de la especificidad de la disciplina da lugar a una configuración compleja del desarrollo laboral del diseñador. Esto, a partir de la coexistencia de postulados que sostienen, por un lado, la tradicional práctica de diseño de producto como aquella ciertamente específica de la disciplina, y por otro, la pluralidad de áreas de inserción, como atributo del diseñador industrial, en tanto 'prestador' de servicios diversos para el mercado y la sociedad. Con respecto a esto, y principalmente en referencia a la mentada legitimidad de la disciplina en

espaces de légitimation des savoirs et compétentes associés aux identités." (Traducción de la autora). 
el área de diseño de productos, que instala la necesidad de un discurso único en torno a la orientación de los perfiles diferenciados, algunos egresados sostienen que:

“...Es que justamente esa falta de definiciones también es lo que enriquece la profesión, ¿no? O sea, que uno puede formarse y en realidad abrir el campo hacia donde vos... Digamos, son herramientas... Me parece que más que una profesión es como una formación que te da herramientas para meterte en el campo... O sea, en un terreno de producción, pero que sea de producción de producto, o sea, ya es como muy amplio. Y estaría re bueno que desde la Facultad también se promueva que esto es así, ¿no? Que no se dé una visión de "hay una imagen del diseñador industrial que es ésta", esto del núcleo chiquito, ¿no? en...[diseño de producto] que después genera también mucha frustración." (Sandra, diseñadora en estudio independiente).

“...O sea, una cosa es lo que a uno le enseñan, otra cosa es lo que termina haciendo, entonces si es tan diferente hay que tratar de coordinarlo para que los diseñadores aprendan lo que van a hacer después por lo menos, o ver qué es lo que pide el mercado, a ver si dentro de lo que pide el mercado les gusta algo y se pueden dedicar a eso. Y no salir con unos humos porque hacen... en la Facultad los trabajos es de diseñar computadoras, diseñás electrodomésticos, y después para diseñar en la vida real es imposible, no sé cuánta gente diseña electrodomésticos en este país, deben ser cinco diseñadores." (Santiago, trabaja en empresa).

Aquí se evidencia la necesidad de articular discursos impartidos institucionalmente sobre el perfil con el que se forma al alumno, con las prácticas profesionales a desarrollar posteriormente. En este sentido, si bien la carrera se ha orientado hacia una apertura en sentido de una ampliación de la formación, aún 
la visión que predomina en relación a su inserción laboral se encuentra definida, dada la legitimidad -y la fuerza- de la tradición, en términos de Williams (2000), por la práctica del diseño de productos, históricamente reconocida en tanto especificidad del diseñador industrial.

En este punto, la necesidad de articular aquello que se performa como identidad para otro, "lo que el otro dice que uno es", en este caso, el profesional en el que el diseñador deviene -institucionalmente percibido como potencial 'diseñador de productos'con "lo que uno dice de sí mismo" o "lo que uno mismo dice ser" -que define a este profesional en torno a una figura más compleja que un diseñador de productos- se vuelve clara. Esta desarticulación que se genera entre lo 'heredado' y lo 'proyectado', y mismo, entre lo 'heredado' y lo 'real', puede generar "frustración" entre los egresados, tal como planteaba la entrevistada anteriormente. Esto es, se produce una ruptura, un desencuentro entre ambas dimensiones -la imagen 'ideal' de inserción y la 'real'- al volverse "imposible", tal como expresaba un entrevistado, la inserción en función del postulado tradicional del diseño de productos, conduciendo al diseñador a 'rearmar' su figura, su identidad, sobre la base de estas instancias desencontradas. En este sentido, en términos de Dubar, se plantea la idea de continuidad o ruptura a la que puede conducir la transacción subjetiva en el proceso de construcción identitaria:

La transacción subjetiva puede conducir a una continuidad entre identidad heredada e identidad pretendida o a una ruptura, a un desfasaje entre la definición de sí resultante de su trayectoria anterior y la proyección de sí en el futuro. (...) Las identidades construidas sobre el modo de la ruptura implican una dualidad entre dos espacios y una imposibilidad de construirse una identidad en el futuro al interior del espacio productor de su identidad pasada. Para encontrar o reencontrar una identidad, es necesario cambiar de espacio. La identidad proyectada 
puede ser sobrevalorizada o desvalorizada en relación a la identidad heredada, ella está en ruptura con ésta. (2000: 236) $)^{13}$.

Tal como señaláramos, el desencuentro generado entre la identidad 'tradicional', aquella avalada por la Universidad, basada en el postulado moderno del diseñador abocado al diseño de productos, constituida en tanto identidad 'heredada' -una de las posibles identidades heredadas que el sujeto puede construir para sí-, no se corresponde con aquella 'proyectada' por el diseñador en su propio devenir. Este desencuentro, planteado por Dubar, en términos de ruptura resultante de la transacción subjetiva, vuelve necesario construir una identidad por fuera de este espacio, al interior del cual se construyó y legitimó la identidad pasada. En este sentido, es posible pensar que la demanda proveniente de los alumnos a fin de ser formados bajo nuevos perfiles, en relación al caso de la autoproducción, haya constituido en sí un nuevo espacio generador de una identidad proyectada, divergente de la 'tradicional' o 'heredada'. Pero en la medida en que persista el discurso que legitime institucionalmente esta identidad, en tanto pasada, heredada, aquella proyectada seguirá en ruptura con ésta, desencontrándose, así, estos espacios: uno, propio de la identidad performada históricamente; otro, conformado en relación al devenir y al nuevo contexto que da lugar a la identidad proyectada, tornando la figura profesional, ciertamente imprecisa.

Con respecto a esto, cabe mencionar aquello que planteaba una entrevistada, quien expresaba que: “...entre la demanda y

13 Texto original: "La transaction subjective peut aboutir à une continuité entre identité héritée et identité visée ou à une rupture, à un décalage, entre la définition de soi issue de sa trajectoire antérieure et la projection de soi dans l'avenir. (...) Les identités construites sur le mode de la rupture impliquent une dualité entre deux espaces et une impossibilité de se construire une identité d'avenir à l'interieur de l'espace producteur de son identité passée. Pour trouver ou retrouver une identité, il faut changer d'espace. L'identité projetée peut être survalorisée ou dévalorisée par rapport à l'identité héritée, elle est en rupture avec elle." (Traducción de la autora). 
lo que nosotros hacemos hay por ahí un hueco...", al dejar en claro la distancia entre la diversidad de acción para la cual los mismos son formados, a partir de una multiplicidad de herramientas -pero aún portando la especificidad nominal del 'diseñador de productos'-, y la demanda de estos profesionales, aún vinculada a cierto desconocimiento sobre su propia labor, o, en casos de conocimiento, vinculada a una demanda específica de diseñador de producto. Esta cuestión refleja sin dudas una de las principales dificultades que debe atravesar el diseñador en su trayectoria profesional: el devenir constitutivo del diseñador industrial al interior de un entramado en el cual confluyen prácticas y discursos en un punto divergentes, en un punto desencontrados. Esto da lugar, entonces, a un proceso de construcción de identidad sobre la base de desencuentros o rupturas, que se dejan entrever en los mismos relatos de los entrevistados, a modo de evidenciar discursivamente el dilema que atraviesa su propia identidad y reconocimiento.

\section{Conclusiones}

Diversas cuestiones han sido analizadas en este trabajo, a fin de no sólo buscar desentrañar cuestiones inherentes a la construcción de la identidad profesional del diseñador industrial, sino también de poner en escena las dificultades propias que atraviesan la trama de una inserción laboral compleja.

En este sentido, en la medida que pudimos poner en escena divergencias entre discursos performativos que sostienen un perfil 'industrialista' y aquellos que sostienen la redefinición disciplinar en función de una mayor diversidad, y a partir de pensar la contradicción entre aquello heredado sobre lo que estos profesionales cimientan sus propios saberes, y aquello anhelado para sí, es que pudimos comprender la dificultad que atraviesa esta figura, y la complejidad que ésta encuentra en términos de poder asumir una identidad constitutiva. Esta contradicción o desarticulación entre lo que alguna vez el diseñador industrial 
fue y lo que hoy es, es lo que les genera a estos profesionales una ruptura con su propia historia. Esto, en el sentido de que se perciben a sí mismos -y los perciben- como diseñadores industriales, pero al contemplar deseos y proyecciones propias, se sienten o se proyectan como diseñadores "múltiples", convocados por una realidad más amplia que la propiamente industrial. El diseñador industrial deviene, en un punto, no industrial, y es, en este mismo entramado simbólico, en el que debe construir su nueva identidad, que integre, sin rupturas, aquello a lo que aspira ser y esto que, en un punto, le es dado.

A su vez, la mayor visibilidad alcanzada por la práctica autogestionada en los últimos años ubica al diseñador como un actor reconocido socialmente, pero que aún debe precisar en mayor medida su rol en el mercado. Esto, justamente, dado que se trata de un reconocimiento que no llega a dar cuenta del completo potencial de este profesional, al reconocer una dimensión del mismo: la práctica de la autoproducción. Con lo cual, el hecho de reconocer al diseñador en relación a este perfil, y sólo a éste, implica desconocer las amplias competencias que lo integran. Este reduccionismo limita no sólo el reconocimiento, sino la propia valoración de las competencias profesionales.

Ante esto, nos preguntamos: ¿Cómo pensarse desde un Nosotros cuando el Yo es lo que prevalece? Ante la dominación de una práctica autogestionada, la connotación subjetiva es la que impera, con lo cual el pensarse como parte de un colectivo constituye ciertamente otra dificultad, que busca en cierta formar ser superada al 'acercarse' a otras figuras que sí detentan mayor cohesión como grupo profesional. Pero, ante la integridad del otro diferente (arquitectos, ingenieros), se diluye la figura diversa del diseñador. En este escenario, la desarticulación entre el Nosotros y el Yo, acentúa su débil construcción identitaria. Con lo cual nos planteamos: ¿cómo constituirse colectivamente ante una diversidad y diferenciación inherentes? Es esta misma diversidad, el encontrarse ciertamente diferenciado del otro generalizado, lo que 
afecta el pensarse a sí mismos como colectivo. En este punto podemos decir que la diversidad es inherente a la profesión, y la individualidad también. El 'Nosotros' aún debe percibirse, y sentirse, como tal. El desafío será alcanzar, colectivamente, esta meta.

\section{Bibliografía}

Battistini, Osvaldo (2009): "Ser estable: ¿una necesidad en las construcciones identitarias?". En Battistini, O.; Bialakowsky, A.; Busso, M. y Costa, M.I. (Comps.). Los trabajadores en la nueva época capitalista. Entre el ser y el saber. Buenos Aires, Teseo.

Correa, María Eugenia (2010): El fenómeno del Diseño independiente en la Ciudad de Buenos Aires: Análisis de la conformación de un nuevo trabajador cultural en la escena local. Tesis de Maestría no publicada. Buenos Aires, Instituto de Altos Estudios Sociales, Universidad de San Martín.

Cuche, Denys (1999): “Cultura e identidad". En Cuche, Denys, La noción de cultura en las ciencias sociales. Buenos Aires, Nueva visión.

Chiapponi, Medardo (1999): Cultura social del producto. Nuevas fronteras para el diseño industrial. Buenos Aires, Infinito.

Devalle, Verónica (2009): La travesía de la forma. Emergencia y consolidación del diseño gráfico (1948-1984). Buenos Aires, Paidós.

Diccionario Enciclopédico Abreviado, Espasa-Calpe S.A. (1986), Segunda Edición, Apéndice III, Madrid.

Dubar, Claude (2002): La crisis de las identidades. La interpretación de una mutación. Barcelona, Bellaterra.

(2001): "El trabajo y las identidades profesionales y personales". En Revista Latinoamericana de Estudios del Trabajo, "Trayectorias ocupacionales y mercado de trabajo", Año 7, N 13. Buenos Aires.

------ (2000): La socialisation, construction des identités sociales et professionnelles. París, Armand Colin.

Dubar, Claude, Tripier, Pierre (1998): Sociologie des professions. París, Armand Colin.

Fiell, Charlotte y Fiell, Peter (2001): Diseño del Siglo XX. Colonia, Taschen.

Heskett, John (2002): El diseño en la vida cotidiana. Barcelona, Gustavo Gili. 
Longo, María Eugenia (2003): “Representaciones sociales en torno al trabajo e identidad en varones pobres". 6to Congreso Nacional de Estudios del Trabajo. ASET (Asociación Argentina de Especialistas en Estudios del Trabajo). Buenos Aires. [En línea] Disponible en: http:/ /www.aset.org.ar/congresos/6/archivosPDF/grupoTematico04/023.pdf

Machuca Barbosa, Adriana Elizabeth (2008): La identidad profesional de los sociólogos. Tesis de Maestría en Ciencias Sociales. FLACSO. México. [En línea] Disponible en: http://conocimientoabierto.flacso.edu.mx/medios/tesis/machuca_ae.pdf

Maldonado, Tomás (1993): El Diseño Industrial reconsiderado. Barcelona, Gustavo Gili.

Sainsaulieu, Renaud (1988): L'identité au travail. París, Presses de Sciences Po.

Taylor, Charles (1998): Les sources du moi. La formation de l'identité moderne. París, Seuil.

------ (1999): “Identidad y reconocimiento". En Revista Internacional de Filosofía Política. Madrid, UNED-Universidad Autónoma Metropolitana.

Williams, Raymond (2000): Marxismo y literatura. Barcelona, Península/ Biblos. 